\title{
Finite Groups with Weakly $s$-Semipermutable Subgroups
}

\author{
Changwen Li
}

\begin{abstract}
Suppose $G$ is a finite group and $H$ is a subgroup of $G$. $H$ is said to be $s$ semipermutable in $G$ if $H G_{p}=G_{p} H$ for any Sylow $p$-subgroup $G_{p}$ of $G$ with $(p,|H|)=1 ; H$ is called weakly $s$-semipermutable in $G$ if there is a subgroup $T$ of $G$ such that $G=H T$ and $H \cap T$ is $s$-semipermutable in $G$. We investigate the influence of weakly $s$-semipermutable subgroups on the structure of finite groups. Some recent results are generalized and unified.
\end{abstract}

\section{Introduction.}

All groups considered in this paper will be finite. We use conventional notions and notation, as in Huppert [1]. $G$ denotes always a group, $|G|$ is the order of $G, \pi(G)$ denotes the set of all primes dividing $|G|$ and $G_{p}$ is a Sylow $p$-subgroup of $G$ for some $p \in \pi(G)$. Let $\mathcal{F}$ be a class of groups. We call $\mathcal{F}$ a formation provided that (i) if $G \in \mathcal{F}$ and $H \triangleleft G$, then $G / H \in \mathcal{F}$, and (ii) if $G / M$ and $G / N$ are in $\mathcal{F}$, then $G /(M \cap N)$ is in $\mathcal{F}$ for any normal subgroups $M, N$ of $G$. A formation $\mathcal{F}$ is said to be saturated if $G / \Phi(G) \in \mathcal{F}$ implies that $G \in \mathcal{F}$. In this paper, $\mathcal{U}, \mathcal{N}$ will denote the class of all supersolvable groups and the class of all nilpotent groups, respectively. As wellknown results, $\mathcal{U}, \mathcal{N}$ are saturated formations.

Two subgroups $H$ and $K$ of $G$ are said to be permutable if $H K=K H$. A subgroup $H$ of $G$ is said to be $s$-permutable (or $s$-quasinormal, $\pi$ quasinormal) in $G$ if $H$ permutes with every Sylow subgroup of $G$ [2]. Asaad, Ramadan and Shaalan proved in [3]: Suppose $G$ is a solvable group with a normal subgroup $H$ such that $G / H$ is supersolvable. If all

(*) Indirizzo dell'A.: School of Mathematical Science, Xuzhou Normal University, Xuzhou, 221116, China.

E-mail: lcwxz@xznu.edu.cn

The project is supported by the Natural Science Foundation of China (No:11071229) and the Natural Science Foundation of the Jiangsu Higher Education Institutions (No:10KJD110004). 
maximal subgroups of any Sylow subgroup of $F(H)$ are $s$-permutable in $G$, then $G$ is supersolvable. Later Asaad in [4] extended the result using formation theory: Let $\mathcal{F}$ be a saturated formation containing $\mathcal{U}$. Suppose that $G$ is a solvable group with a normal subgroup $H$ such that $G / H \in \mathcal{F}$. If all maximal subgroups of all Sylow subgroups of $F(H)$ are $s$-permutable in $G$, then $G \in \mathcal{F}$. As a generalization of s-permutable subgroup, the concept of $s$-semipermutable subgroup is introduced. A subgroup $H$ of $G$ is said to be $s$-semipermutable in $G$ if $H G_{p}=G_{p} H$ for any Sylow $p$ subgroup $G_{p}$ of $G$ with $(p,|H|)=1$. Q. Zhang and L. Wang [5] obtained the following: Let $\mathcal{F}$ be a saturated formation containing $\mathcal{U}$. Suppose that $G$ is a group with a solvable normal subgroup $H$ such that $G / H \in \mathcal{F}$. If all maximal subgroups of all Sylow subgroups of $F(H)$ are $s$-semipermutable in $G$, then $G \in \mathcal{F}$. In recent years, it has been of interest to use supplementation properties of subgroups to characterize properties of a group. For example, Y. Wang [6] introduced the concept of $c$-supplemented subgroups and obtained the similar result in [7]: Let $\mathcal{F}$ be a saturated formation containing $\mathcal{U}$. Suppose that $G$ is a group with a solvable normal subgroup $H$ such that $G / H \in \mathcal{F}$. If all maximal subgroups of all Sylow subgroups of $F(H)$ are $c$-supplemented in $G$, then $G \in \mathcal{F}$.

There is no obvious general relationship between $s$-semipermutable subgroup and $c$-supplemented subgroup. Hence it is meaningful to unify and generalize the two concepts and relate results. Recall that $H$ is $c$ supplemented in $G$ if there exists a subgroup $K_{1}$ such that $G=H K_{1}$ and $H \cap K_{1} \leq H_{G}$, where $H_{G}$ is the maximal normal subgroup of $G$ contained in $H$. In this case, writing $K=H_{G} K_{1}$ we have $G=H K$ and $H \cap K=H_{G}$; of course, $H \cap K$ is $s$-semipermutable in $G$. On the basis of this observation, we introduce a new embedding property:

DeFinition 1.1. A subgroup $H$ of a group $G$ is called weakly s-semipermutable in $G$ if there is a subgroup $T$ of $G$ such that $G=H T$ and $H \cap T$ is s-semipermutable in $G$.

In the present paper, we study the influence of weakly $s$-semipermutable subgroups on the structure of some groups. In particular, we give some new characterizations of supersolvability and $p$-nilpotency of a group (and, more general, a group belonging to a given formation of finite groups) by using the weakly $s$-semipermutability of some primary subgroups. As application, we unify and generalize a series of known results. 


\section{Preliminaries.}

Lemma 2.1. Suppose that $H$ is an s-semipermutable subgroup of a group $G$ and $N$ is a normal subgroup of $G$. Then

(a) $H$ is s-semipermutable in $K$ whenever $H \leq K \leq G$.

(b) If $H$ is p-group for some prime $p \in \pi(G)$, then $H N / N$ is s-semipermutable in $G / N$.

(c) If $H \leq O_{p}(G)$, then $H$ is s-permutable in $G$.

Proof. (a) is [5, Property 1], (b) is [5, Property 2], and (c) is [5, Lemma 3].

LEMMA 2.2. Let $U$ be a weakly s-semipermutable subgroup of a group $G$ and $N$ a normal subgroup of $G$. Then

(a) If $U \leq H \leq G$, then $H$ is weakly s-semipermutable in $H$.

(b) Suppose that $U$ is a p-group for some prime $p$. If $N \leq U$, then $U / N$ is weakly s-semipermutable in $G / N$.

(c) Suppose $U$ is a p-group for some prime $p$ and $N$ is a $p^{\prime}$-subgroup, then $U N / N$ is weakly s-semipermutable in $G / N$.

Proof. By the hypotheses, there is a subgroup $K$ of $G$ such that $G=U K$ and $U \cap K$ is $s$-semipermutable in $G$.

(a) $H=H \cap U K=U(H \cap K)$ and $U \cap(H \cap K)=U \cap K$ is s-semipermutable in $H$ by Lemma 2.1(a). Hence $U$ is weakly $s$-semipermutable in $H$.

(b) $G / N=U K / N=U / N \cdot N K / N$ and $(U / N) \cap(K N / N)=(U \cap K N) / N=$ $(U \cap K) N / N$ is $s$-semipermutable in $G / H$ by Lemma $2.1(b)$. Hence $U / N$ is weakly $s$-semipermutable in $G / N$.

(c) Since $(|G: K|,|N|)=1, N \leq K$. It is easy to see that $G / N=$ $U N / N \cdot K N / N=U N / N \cdot K / N$ and $(U N / N) \cap(K / N)=(U N \cap K) / N=$ $(U \cap K) N / N$ is $s$-semipermutable in $G / N$ by Lemma 2.1(b). Hence $U N / N$ is weakly $s$-semipermutable in $G / N$.

LemMa 2.3 ([8], Lemma 2.6). Let $H$ be a solvable normal subgroup of a group $G(H \neq 1)$. If every minimal normal subgroup of $G$ which is contained in $H$ is not contained in $\Phi(G)$, then the Fitting subgroup $F(H)$ of $H$ is the direct product of minimal normal subgroups of $G$ which are contained in $H$.

Lemma 2.4 ([7], Lemma 2.8). Let $M$ be a maximal subgroup of $G, P$ a normal p-subgroup of $G$ such that $G=P M$, where $p$ a prime. Then $P \cap M$ is a normal subgroup of $G$. 
LEMMA 2.5 ([9], Lemma 2.16). Let $\mathcal{F}$ be a saturated formation containing $\mathcal{U}$. Suppose that $G$ is a group with a normal subgroup $N$ such that $G / N \in \mathcal{F}$. If $N$ is cyclic, then $G \in \mathcal{F}$.

Lemma 2.6 ([9], Lemma 2.20). Let $A$ be a $p^{\prime}$-automorphisms of a $p$ group $P$, where $p$ is an odd prime. Assume that every subgroup of $P$ with prime order is A-invariant. Then $A$ is cyclic.

Lemma 2.7 ([1], III, 5.2 and IV, 5.4). Suppose G is a group which is not nilpotent but whose proper subgroups are all nilpotent. Then

(a) G has a normal Sylow p-subgroup $P$ for some prime $p$ and $G=P Q$, where $Q$ is a non-normal cyclic $q$-subgroup for some prime $q \neq p$.

(b) $P / \Phi(P)$ is a minimal normal subgroup of $G / \Phi(P)$.

(c) If $P$ is non-abelian and $p>2$, then the exponent of $P$ is $p$; If $P$ is non-abelian and $p=2$, then the exponent of $P$ is 4 .

(d) If $P$ is abelian, then the exponent of $P$ is $p$.

(e) $\Phi(P) \leq Z(P)$.

Lemma 2.8 ([8], Lemma 3.12). Let $P$ be a Sylow p-subgroup of a group $G$, where $p$ is the smallest prime dividing $|G|$. If $G$ is $A_{4}$-free and $|P| \leqslant p^{2}$, then $G$ is p-nilpotent.

\section{Results.}

THEOREM 3.1. Let $\mathcal{F}$ be a saturated formation containing $\mathcal{U}$. A group $G \in \mathcal{F}$ if and only if there is a normal subgroup $E$ of $G$ such that $G / E \in \mathcal{F}$ and every cyclic subgroup $\langle x\rangle$ of any noncyclic Sylow subgroup of $E$ with prime order or order 4 (if the Sylow 2-subgroup is non-abelian) is weakly s-semipermutable in $G$.

Proof. We need only to prove the sufficiency part since the necessity part is evident. Suppose that the assertion is false and let $(G, E)$ be a counterexample for which $|G||E|$ is minimal. Then

(1) $E$ is solvable.

Let $K$ be any proper subgroup of $E$. Then $|K|<|G|$ and $K / K \in \mathcal{U}$. Let $\langle x\rangle$ be any cyclic subgroup of any noncyclic Sylow subgroup of $K$ with prime order or order 4 ( if the Sylow 2-subgroup is non-abelian ). It is clear that $\langle x\rangle$ is also a cyclic subgroup of a noncyclic Sylow subgroup of $E$ with prime order or order 4 . By the hypothesis, $\langle x\rangle$ is weakly $s$-semipermutable 
in $G$. By Lemma 2.2, $\langle x\rangle$ is weakly $s$-semipermutable in $K$. This shows that the hypothesis still holds for $(\mathcal{U}, K)$. By the choice of $G, K$ is supersolvable. By [10, Theorem 3.11.9], $E$ is solvable.

(2) $G^{\mathcal{F}}$ is a $p$-group,where $G^{\mathcal{F}}$ is the $\mathcal{F}$-residual of $G . G^{\mathcal{F}} / \Phi\left(G^{\mathcal{F}}\right)$ is a chief factor of $G$ and $\exp \left(G^{\mathcal{F}}\right)=p$ or $\exp \left(G^{\mathcal{F}}\right)=4$ (if $p=2$ and $G^{\mathcal{F}}$ is non-abelian).

Since $G / E \in \mathcal{F}, G^{\mathcal{F}} \leq E$. Let $M$ be a maximal subgroup of $G$ such that $G^{\mathcal{F}} \nsubseteq M$ (that is, $M$ is an $\mathcal{F}$-abnormal maximal subgroup of $G$ ). Then $G=M E$. We claim that the hypothesis holds for $(\mathcal{F}, M)$. In fact, $M / M \cap E \cong M E / E=G / E \in \mathcal{F}$ and by the similar argument as above, we can prove that the hypothesis holds for $(\mathcal{F}, M)$. By the choice of $G, M \in \mathcal{F}$. Thus (2) holds by [10, Theorem 3.4.2].

(3) $\langle x\rangle$ is $s$-permutable in $G$ for any element $x \in G^{\mathcal{F}}$.

Let $x \in G^{\mathcal{F}}$. Then the order of $x$ is $p$ or 4 by step (2). By the hypothesis, $\langle x\rangle$ is weakly $s$-semipermutable in $G$. Then there is a group $T$ of $G$ such that $G=\langle x\rangle T$ and $\langle x\rangle \cap T$ is $s$-semipermutable in $G$. It follows that $G=\langle x\rangle T$ and $G^{\mathcal{F}}=G^{\mathcal{F}} \cap G=G^{\mathcal{F}} \cap\langle x\rangle T=\langle x\rangle\left(G^{\mathcal{F}} \cap T\right)$. Since $G^{\mathcal{F}} / \Phi\left(G^{\mathcal{F}}\right)$ is abelian, $\left(G^{\mathcal{F}} \cap T\right) \Phi\left(G^{\mathcal{F}}\right) / \Phi\left(G^{\mathcal{F}}\right) \triangleleft G / \Phi\left(G^{\mathcal{F}}\right)$. Since $G^{\mathcal{F}} / \Phi\left(G^{\mathcal{F}}\right)$ is a chief factor of $G$, $G^{\mathcal{F}} \cap T \leq \Phi\left(G^{\mathcal{F}}\right)$ or $G^{\mathcal{F}}=\left(G^{\mathcal{F}} \cap T\right) \Phi\left(G^{\mathcal{F}}\right)=G^{\mathcal{F}} \cap T$. If $G^{\mathcal{F}} \cap T \leq \Phi\left(G^{\mathcal{F}}\right)$, $\langle x\rangle=G^{\mathcal{F}} \triangleleft G$. In this case, $\langle x\rangle$ is $s$-permutable in $G$. Now assume that $G^{\mathcal{F}}=G^{\mathcal{F}} \cap T$. Then $T=G$ and $\langle x\rangle=\langle x\rangle \cap T$ is $s$-semipermutable in $G$. Since $\langle x\rangle \leq G^{\mathcal{F}} \leq O_{p}(G),\langle x\rangle$ is $s$-permutable in $G$ by Lemma 2.1 .

(4) $\left|G^{\mathcal{F}} / \Phi\left(G^{\mathcal{F}}\right)\right|=p$.

Assume that $\left|G^{\mathcal{F}} / \Phi\left(G^{\mathcal{F}}\right)\right| \neq p$ and let $L / \Phi\left(G^{\mathcal{F}}\right)$ be any cyclic subgroup of $G^{\mathcal{F}} / \Phi\left(G^{\mathcal{F}}\right)$. Let $x \in L \backslash \Phi\left(G^{\mathcal{F}}\right)$. Then $L=\langle x\rangle \Phi\left(G^{\mathcal{F}}\right)$. Since $\langle x\rangle$ is $s$-permutable in $G$ by step (3), $L / \Phi\left(G^{\mathcal{F}}\right)$ is $s$-permutable in $G / \Phi\left(G^{\mathcal{F}}\right)$. It follows from [9, Lemma 2.11] that $G^{\mathcal{F}} / \Phi\left(G^{\mathcal{F}}\right)$ has a maximal subgroup which is normal in $G / \Phi\left(G^{\mathcal{F}}\right)$. But this is impossible since $G^{\mathcal{F}} / \Phi\left(G^{\mathcal{F}}\right)$ is a chief factor of $G$. Thus $\left|G^{\mathcal{F}} / \Phi\left(G^{\mathcal{F}}\right)\right|=p$.

(5) The final contradiction.

Since $\left(G / \Phi\left(G^{\mathcal{F}}\right)\right) /\left(G^{\mathcal{F}} / \Phi\left(G^{\mathcal{F}}\right)\right) \cong G / G^{\mathcal{F}} \in \mathcal{F}, G / \Phi\left(G^{\mathcal{F}}\right) \in \mathcal{F}$ by Lemma 2.5. As $\Phi\left(G^{\mathcal{F}}\right) \leq \Phi(G)$ and $\mathcal{F}$ is a saturated formation, we have $G \in \mathcal{F}$. The final contradiction completes the proof.

Corollary 3.2 [14], Theorem 3.4). Let $\mathcal{F}$ be a saturated formation containing $\mathcal{U}$, the class of all supersolvable groups. If every cyclic subgroup of $G^{\mathcal{F}}$ with prime order or order 4 is c-normal in $G$, then $G \in \mathcal{F}$.

Corollary 3.3 ([15], Theorem 4.2). If every cyclic subgroup of a group $G$ with prime order or order 4 is c-normal in $G$, then $G$ is supersolvable. 
CoRollary 3.4 ([16], Theorem 4.1). If every cyclic subgroup of $G^{\mathcal{U}}$ with prime order or order 4 is c-supplemented in $G$, then $G$ is supersolvable.

CoRollary 3.5 ([17], Theorem 1). Let $\mathcal{F}$ be a saturated formation containing $\mathcal{U}$, the class of all supersolvable groups. If there is a normal subgroup $H$ of $G$ such that $G / H \in \mathcal{F}$ and every cyclic subgroup of $H$ with prime order or order 4 is s-permutable in $G$, then $G \in \mathcal{F}$.

COROLlaRY 3.6 ([21], Theorem 3.9). Let $\mathcal{F}$ be a saturated formation containing $\mathcal{U}$, the class of all supersolvable groups. Then $G \in \mathcal{F}$ if and only if there is a normal subgroup $H$ of $G$ such that $G / H \in \mathcal{F}$ and the subgroups prime order or order 4 of $H$ with are c-normal in $G$.

CoRollary 3.7 ([19], Theorem 3.1). Let $G$ be a group and $N$ a normal subgroup of a group $G$ such that $G / N$ is supersolvable. If every minimal subgroup of $E$ is c-supplemented in $G$ and if every cyclic subgroup of order 4 of $N$ is c-normal in $G$, then $G$ is supersolvable.

THEOREM 3.8. Let $\mathcal{F}$ be a saturated formation containing $\mathcal{U}$. A group $G \in \mathcal{F}$ if and only if there is a solvable normal subgroup $H$ of $G$ such that $G / H \in \mathcal{F}$ and every cyclic subgroup $\langle x\rangle$ of any noncyclic Sylow subgroup of $F(H)$ with prime order or order 4 (if the Sylow 2-subgroup is non-abelian) is weakly s-semipermutable in $G$.

Proof. It is clear that the condition is necessary. We only need to prove that it is sufficient. Suppose that the assertion is false and let $(G, H)$ be a counterexample for which $|G||H|$ is minimal. Let $p$ be the smallest prime divisor of $|F(H)|$ and $P$ the Sylow $p$-subgroup of $F(H)$. Then $P \triangleleft G$. Now we proceed with our proof as follows:

(1) $F(H) \neq H$ and $C_{H}(F(H)) \leq F(H)$.

If $F(H)=H$, then $G \in \mathcal{F}$ by Theorem 3.1, a contradiction. Obviously, $C_{H}(F(H)) \leq F(H)$ since $H$ is solvable.

(2) Let $V / P=F(H / P)$ and $Q$ be a Sylow $q$-subgroup of $V$, where $q \| V / P \mid$. Then $q \neq p$ and either $Q \leq F(H)$ or $p>q$ and $C_{Q}(P)=1$.

Since $V / P$ is nilpotent, $Q P / P$ char $V / P$ and so $Q P \triangleleft H$. Then, it is easy to see that $p \neq q$. By Theorem 3.1, $P Q$ is supersolvable. If $q>p$, then $Q \triangleleft P Q$ and so $Q \leq F(H)$. Now assume that $p>q$. Then $p>2$. Since $p$ is the minimal prime divisor of $|F(H)|, F(H)$ is a $q^{\prime}$-group. Let $R$ be a Sylow $r$ - 
subgroup of $F(H)$ where $r \neq p$. Then $r \neq q$ and so $[R, Q] \leq P$. Assume that for some $x \in Q$, we have $x \in C_{H}(P)$. Since $V / P$ is nilpotent, $[R,\langle x\rangle]=$ $[R,\langle x\rangle,\langle x\rangle]=1$ by $\left[11\right.$, Chapter 5 , Theorem 3.6]. Hence $x \in C_{H}(F(H))$. By (1), $C_{H}(F(H)) \leq F(H)$ and so $C_{Q}(P)=1$.

(3) $p>2$. If $p=2$, then by (2), we see that $F(H / P)=F(H) / P$ and $2 \nmid|F(H / P)|$. This implies that if $\langle x\rangle P / P$ is an arbitrary minimal subgroup of $F(H) / P$, then $|x|=r$, where $r \neq 2$. By Lemma 2.2, every minimal subgroup of $F(H / P)$ is weakly $s$-semipermutable in $G / P$. Hence $(G / P, H / P)$ satisfies the hypothesis. The minimal choice of $(G, H)$ implies that $G / P \in \mathcal{F}$. Hence by Theorem 3.1, $G \in \mathcal{F}$, a contradiction. Thus, (3) holds.

(4) Final contradiction. Let $V / P=F(H / P)$ and $Q$ be a Sylow $q$-subgroup of $V$, where $q \| V / P \mid$. Then by (2), either $Q \leq F(H)$ or $p>q$ and $C_{Q}(P)=1$. In the second case, $Q$ is cyclic by (3) and Lemma 2.6. Hence every Sylow subgroup of $F(H / P)$ either is cyclic or is contained in $F(H)$. Moreover by (2), $p \nmid|F(H / P)|$. Let $K / P$ be a cyclic subgroup of a non-cyclic Sylow subgroup of $F(H / P)$ with prime order. Then it is easy to see that $K / P=\langle x\rangle P / P$, where $\langle x\rangle$ is a cyclic subgroup of some non-cyclic Sylow subgroup of $F(H)$ with prime order. By hypothesis, $\langle x\rangle$ is weakly $s$-semipermutable in $G$. Hence $\langle x\rangle P / P$ is weakly $s$-semipermutable in $G / P$ by Lemma 2.2. This shows that $(G / P, H / P)$ satisfies the hypothesis. The minimal choice of $(G, H)$ implies that $G / P \in \mathcal{F}$. Therefore, $G \in \mathcal{F}$ by Theorem 3.1. The final contradiction completes the proof.

Corollary 3.9 ([22], Theorem 3). Let $G$ be a group and E a solvable normal subgroup of $G$ such that $G / E$ is supersolvable. If all minimal subgroups and all cyclic subgroups with order 4 of $F(E)$ are c-normal in $G$, then $G$ is supersolvable.

CoRollary 3.10 ([23], Theorem 2). Let $\mathcal{F}$ be a saturated formation containing $\mathcal{U}$. Suppose that $G$ is a group with a solvable normal subgroup $H$ such that $G / H \in \mathcal{F}$. If all minimal subgroups and all cyclic subgroups with order 4 of $F(H)$ is c-normal in $G$, then $G \in \mathcal{F}$.

COROLLARY 3.11 ([24], Theorem 3). Let $\mathcal{F}$ be a saturated formation containing $\mathcal{U}$. A group $G \in \mathcal{F}$ if and only if there is a solvable normal subgroup $H$ of $G$ such that $G / H \in \mathcal{F}$ and the subgroups of prime order or order 4 of $F(H)$ is c-normal in $G$.

COROLLARY 3.12 ([7], Theorem 4.1). Let $\mathcal{F}$ be a saturated formation containing $\mathcal{U}$. Suppose that $G$ is a group with a solvable normal subgroup 
$H$ such that $G / H \in \mathcal{F}$. If all minimal subgroups and all cyclic subgroups with order 4 of $F(H)$ is c-supplemented in $G$, then $G \in \mathcal{F}$.

Corollary 3.13 ([5], Theorem 4). Let $\mathcal{F}$ be a saturated formation containing $\mathcal{U}$. Suppose that $G$ is a group with a solvable normal subgroup $H$ such that $G / H \in \mathcal{F}$. If all minimal subgroups and all cyclic subgroups with order 4 of $F(H)$ is s-semipermutable in $G$, then $G \in \mathcal{F}$.

Corollary 3.14 ([27], Corollary 1). Suppose G is a solvable group with a normal subgroup $H$ such that $G / H$ is supersolvable. If every subgroup of $F(H)$ of prime order or order 4 is s-permutable in $G$, then $G$ is supersolvable.

Corollary 3.15 ([27], Theorem 1). A group $G \in \mathcal{F}$ if and only if there is a solvable normal subgroup $H$ of $G$ such that $G / H \in \mathcal{F}$ and the subgroups of prime order or order 4 of $F(H)$ is s-permutable in $G$.

THEOREM 3.16. Suppose G is a group. If every subgroup of G with prime order is contained in $Z_{\infty}(G)$ and every cyclic subgroup of $G$ with order 4 is weakly s-semipermutable in $G$ or lies in $Z_{\infty}(G)$, then $G$ is nilpotent.

Proof. Suppose that the theorem is false, and let $G$ be a counterexample of minimal order. Let $H$ be an arbitrary proper subgroup of $G$ and $\langle x\rangle$ be a cyclic subgroup of $H$ with prime order or order 4, then $\langle x\rangle \leq Z_{\infty}(G) \cap H \leq Z_{\infty}(H)$. By Lemma 2.2 , $\langle x\rangle$ is weakly $s$-semipermutable in $H$. Thus $H$ satisfies the hypotheses of the theorem in any case. The minimal choice of $G$ implies that $H$ is nilpotent, thus $G$ is a group which is not nilpotent but whose proper subgroups are all nilpotent. By Lemmas 2.7, $G=P Q$, where $P$ is normal in $G$ for some $p \in \pi(G)$ and $Q$ is non-normal cyclic. Then we have:

(1) $p=2$ and every element with order 4 is weakly $s$-semipermutable in $G$.

If $p>2$, by Lemma 2.7, $\exp (P)=p$. Thus $P \leq Z_{\infty}(G)$ by hypotheses. Therefore, $G / Z_{\infty}(G)$ is nilpotent. It follows that $G$ is nilpotent, a contradiction. If every element with order 4 of $G$ lies in $Z_{\infty}(G)$, then $P \leq Z_{\infty}(G)$, we have the same contradiction. Thus (1) holds.

(2) For every $x \in P \backslash \Phi(P)$, we have $\circ(x)=4$.

If not, there exists $x \in P \backslash \Phi(P)$ and $\circ(x)=2$. Denote $M=\langle x\rangle^{G} \leq P$. Then $M \Phi(P) / \Phi(P) \triangleleft G / \Phi(P)$, we have that $P=M \Phi(P)=M \leq Z(G)$ as $P / \Phi(P)$ is a minimal normal subgroup of $G / \Phi(P)$ by Lemma 2.7, a contradiction. 
(3) Final contradiction.

By (2), every element $x$ in $P \backslash \Phi(P)$ is order 4 . Then $x$ is weakly $s$-semipermutable in $G$. Thus there is a subgroup $T$ of $G$ such that $G=\langle x\rangle T$ and $\langle x\rangle \cap T$ is semipermutable in $G$. Hence $P=P \cap G=P \cap\langle x\rangle T=\langle x\rangle(P \cap T)$. Since $P / \Phi(P)$ is abelian, we have $(P \cap T) \Phi(P) / \Phi(P) \unlhd G / \Phi(P)$. Since $P / \Phi(P)$ is the minimal normal subgroup of $G / \Phi(P), P \cap T \leq \Phi(P)$ or $P=(P \cap T) \Phi(P)=P \cap T$. If $P \cap T \leq \Phi(P)$, then $\langle x\rangle=P$, a contraction with Lemma 2.7(d). Therefore $P=P \cap T$. Then $T=G$ and so $\langle x\rangle$ is $s$ semipermutable in $G$. Since $\langle x\rangle \leq O_{p}(G)=P,\langle x\rangle$ is permutable in $G$ by Lemma 2.1. We have $\langle x\rangle Q$ is a proper subgroup of $G$ and so $\langle x\rangle Q=\langle x\rangle \times Q$. Therefore $x \in N_{G}(Q)$, and it follows that $P \leq N_{G}(Q)$ and $G=P \times Q$, the final contradiction.

THEOREM 3.17. Let $\mathcal{F}$ be a saturated formation such that $\mathcal{N} \subseteq \mathcal{F}$. Let $G$ be a group such that every cyclic subgroup of $G^{\mathcal{F}}$ with order 4 is weakly s-semipermutable in $G$. Then $G \in \mathcal{F}$ if and only if every subgroup of $G^{\mathcal{F}}$ with prime order lies in the $\mathcal{F}$-hypercenter $Z_{\mathcal{F}}(G)$ of $G$.

Proof. If $G \in \mathcal{F}$, then $Z_{\mathcal{F}}(G)=G$ and we are done. So we only need to prove that the converse is true. Assume the converse is false and let $G$ be a counterexample of minimal order. Then $G \notin \mathcal{F}$. Let $x$ be an element with prime order of $G^{\mathcal{F}}$. Then $x \in Z_{\mathcal{F}}(G) \cap G^{\mathcal{F}}$ which is contained in $Z\left(G^{\mathcal{F}}\right)$ by [12, IV, 6.10]. By Lemma 2.2, every cyclic subgroup of $G^{\mathcal{F}}$ with order 4 is weakly $s$-semipermutable in $G^{\mathcal{F}}$. Theorem 3.16 implies that $G^{\mathcal{F}}$ is nilpotent. If $G^{\mathcal{F}} \leq \Phi(G)$, then $G / \Phi(G) \in \mathcal{F}$, hence $G \in \mathcal{F}$ since $\mathcal{F}$ is saturated. This is a contradiction. So there exists a maximal subgroup of $G$, say $M$, such that $G=M G^{\mathcal{F}}=M F(G)$. By [13, Theorem 3.5], we may choose $M$ to be an $\mathcal{F}$ critical maximal subgroup. Since $G / M_{G} \notin \mathcal{F}$, it follows that $Z_{\mathcal{F}}(G) \leq M$. Moreover, a $G$-chief factor $A / B$ below $Z_{\mathcal{F}}(G)$ is actually an $M$-chief factor and $\operatorname{Aut}_{M}(A / B)$ is isomorphic to $\operatorname{Aut}_{G}(A / B)$ because $F(G)$ centralizes $A / B$. Consequently $Z_{\mathcal{F}}(G)$ is contained in $Z_{\mathcal{F}}(M)$. By [12, IV, 1.17], $M^{\mathcal{F}} \leq G^{\mathcal{F}}$. Hence $M$ satisfies the hypotheses of the theorem. The minimal choice of $G$ implies that $M \in \mathcal{F}$. By [10, Theorem 3.4.2], $G$ has the following properties:

(a) $G^{\mathcal{F}}$ is a $p$-group, for some prime $p$.

(b) $G^{\mathcal{F}} / \Phi\left(G^{\mathcal{F}}\right)$ is a minimal normal subgroup of $G / \Phi\left(G^{\mathcal{F}}\right)$.

(c) If $G^{\mathcal{F}}$ is abelian, then $G^{\mathcal{F}}$ is an elementary abelian $p$-group.

(d) If $p>2$, then $\exp \left(G^{\mathcal{F}}\right)=p$; if $p=2$, then $\exp \left(G^{\mathcal{F}}\right)=2$ or 4 .

If $G^{\mathcal{F}}$ is abelian, then $G^{\mathcal{F}}$ is an elementary abelian subgroup by (c). Hence, by hypothesis, we have that $G^{\mathcal{F}} \leq Z_{\mathcal{F}}(G)$. It follows that $G \in \mathcal{F}$. 
This contradiction shows that $G^{\mathcal{F}}$ is nonabelian. If $\exp \left(G^{\mathcal{F}}\right)=p$, then $G^{\mathcal{F}} \leq Z_{\mathcal{F}}(G)$ by hypothesis and consequently $G \in \mathcal{F}$, a contradiction again. Thus, $G^{\mathcal{F}}$ is a non-abelian 2-group and $\exp \left(G^{\mathcal{F}}\right)=4$.

Let $x$ be an arbitrary element of $G^{\mathcal{F}} \backslash \Phi\left(G^{\mathcal{F}}\right)$. Then $|x|=4$. Indeed, suppose that there exists an element $x \in G^{\mathcal{F}} \backslash \Phi\left(G^{\mathcal{F}}\right)$ such that $|x|=2$. Let $T=\langle x\rangle^{G}$. Then $T \leq G^{\mathcal{F}}$ and $T \Phi\left(G^{\mathcal{F}}\right) / \Phi\left(G^{\mathcal{F}}\right)$ is normal in $G / \Phi\left(G^{\mathcal{F}}\right)$. Since $G^{\mathcal{F}} / \Phi\left(G^{\mathcal{F}}\right)$ is a chief factor of $G, G^{\mathcal{F}}=T$, which contradicts the fact that $\exp \left(G^{\mathcal{F}}\right)=4$. Now we will prove $\langle x\rangle$ is $s$-permutable in $G$. By hypothesis, $\langle x\rangle$ is weakly $s$-semipermutable in $G$. Hence there exists a subgroup $K$ of $G$ such that $G=\langle x\rangle K$ and $\langle x\rangle \cap K$ is $s$-semipermutable in $G$. It follows that $G^{\mathcal{F}}=G^{\mathcal{F}} \cap G=G^{\mathcal{F}} \cap\langle x\rangle K=\langle x\rangle\left(G^{\mathcal{F}} \cap K\right)$. Since $G^{\mathcal{F}} / \Phi\left(G^{\mathcal{F}}\right)$ is abelian, $\left(G^{\mathcal{F}} \cap K\right) \Phi\left(G^{\mathcal{F}}\right) / \Phi\left(G^{\mathcal{F}}\right) \triangleleft G / \Phi\left(G^{\mathcal{F}}\right)$. Since $G^{\mathcal{F}} / \Phi\left(G^{\mathcal{F}}\right)$ is a chief factor of $G$, $G^{\mathcal{F}} \cap K \leq \Phi\left(G^{\mathcal{F}}\right)$ or $G^{\mathcal{F}}=\left(G^{\mathcal{F}} \cap K\right) \Phi\left(G^{\mathcal{F}}\right)=G^{\mathcal{F}} \cap K$. If $G^{\mathcal{F}} \cap K \leq \Phi\left(G^{\mathcal{F}}\right)$, $\langle x\rangle=G^{\mathcal{F}} \triangleleft G$. In this case, $\langle x\rangle$ is $s$-permutable in $G$. Now assume that $G^{\mathcal{F}}=G^{\mathcal{F}} \cap K$. Then $K=G$ and $\langle x\rangle$ is $s$-semipermutable in $G$. Since $\langle x\rangle \leq G^{\mathcal{F}} \leq O_{p}(G),\langle x\rangle$ is $s$-permutable in $G$ by Lemma 2.1.

Thus for any $q \in \pi(G), q \neq 2,\langle x\rangle$ is normalized by any Sylow $q$-subgroup $Q$ of $M$. So $Q$ acts on $\langle x\rangle$ by conjugation. But the automorphism group of a cyclic group of order 4 is a cyclic group of order 2 , so $Q$ acts trivially on $\langle x\rangle$ and $Q$ centralizes $\langle x\rangle$. Thus $\langle x\rangle$ is centralized by $O^{2}(M)$, it implies that $G^{\mathcal{F}}$ is centralized by $O^{2}(M)$. Hence $O^{2}(M) \triangleleft G$ as $G=M G^{\mathcal{F}}$. It follows that $G / M_{G}$ is a 2-group. Therefore, $G / M_{G} \in \mathcal{F}$ since $\mathcal{N} \subseteq \mathcal{F}$, a final contradiction. This completes the proof of Theorem 3.17.

CoRollaRY 3.18 ([14], Theorem 3.2). Let $\mathcal{F}$ be a saturated formation such that $\mathcal{N} \subseteq \mathcal{F}$. Let $G$ be a group such that every cyclic subgroup of $G^{\mathcal{F}}$ with order 4 is c-normal in $G$. Then $G \in \mathcal{F}$ if and only if every subgroup of $G^{\mathcal{F}}$ with prime order lies in the $\mathcal{F}$-hypercenter $Z_{\mathcal{F}}(G)$ of $G$.

CoRollaRy 3.19 ([18], Theorem 4.4). Let $\mathcal{F}$ be a saturated formation such that $\mathcal{N} \subseteq \mathcal{F}$. Let $G$ be a group such that every cyclic subgroup of $G^{\mathcal{F}}$ with order 4 is c-supplemented in $G$. Then $G \in \mathcal{F}$ if and only if every subgroup of $G^{\mathcal{F}}$ with prime order lies in the $\mathcal{F}$-hypercenter $Z_{\mathcal{F}}(G)$ of $G$.

Corollary 3.20 ([19], Theorem 2.5). Suppose that $p$ is a prime and $K=G^{\mathcal{N}}$ be the nilpotent residual of $G$. Then $G$ is p-nilpotent if every minimal subgroup of $K$ is contained in $Z_{\infty}(G)$ and every cyclic $\langle x\rangle$ of $K$ with order 4 is c-supplemented in $G$.

Corollary 3.21 ([20], Theorem 2.4). Let $G$ be a finite group and $K=G^{\mathcal{N}}$ be the nilpotent residual of $G$. Then $G$ is nilpotent if and only if 
every minimal subgroup $\langle x\rangle$ of $K$ lies in the hypercenter $Z_{\infty}(G)$ of $G$ and every cyclic element of $P$ with order 4 is c-normal in $G$.

THEOREM 3.22. Let $p$ be the smallest prime dividing the order of a group $G$ and $N$ a normal subgroup of $G$ such that $G / N$ is p-nilpotent. If $G$ is $A_{4}$-free and every subgroup of $N$ with order $p^{2}$ is weakly s-semipermutable in $G$, then $G$ is p-nilpotent.

Proof. Assume that the Theorem is false and let $G$ be a counterexample of minimal order. Then:

(1) Every proper subgroup of $G$ is $p$-nilpotent.

By Lemma 2.8, we see that $|N|_{p}>p^{2}$. Let $L$ be a proper subgroup of $G$. Since $L /(L \cap N) \cong L N / N \leq G / N, L /(L \cap N)$ is $p$-nilpotent. If $|L \cap N|_{p} \leqslant p^{2}$, then $L$ is $p$-nilpotent by Lemma 2.8. If $|L \cap N|_{p}>p^{2}$, then every subgroup of $L \cap N$ of order $p^{2}$ is weakly $s$-semipermutable in $L$ by Lemma 2.2. Hence $L$ is $p$-nilpotent by the choice of $G$. This shows that $G$ is a minimal non- $p$-nilpotent group.

(2) $G$ has the following properties: (i) $G=P Q$, where $P=G^{\mathcal{N}}$ is a normal Sylow $p$-subgroup of $G$ and $Q$ is a non-normal cyclic Sylow $q$-subgroup of $G$; (ii) $P / \Phi(P)$ is a minimal normal subgroup of $G / \Phi(P)$; (iii) If $p>2$, then the exponent of $P$ is $p$; if $p=2$, then the exponent of $P$ is 2 or 4 ; (iv) $\Phi(P) \leq Z(P)$; (v) $p^{3}$ dividing the order of $P$; (vi) $P \leq N$.

By Step (1) and [1, Theorem IV. 5.4], $G$ is a minimal non-nilpotent group. Hence (i)-(iv) follow directly from Lemma 2.7. (v) follows from Lemma 2.8. (vi) is clear since $P=G^{\mathcal{N}}$ is the $p$-nilpotent residual of $G$ and $G / N$ is $p$-nilpotent.

(3) If $H$ is a subgroup of $P$ of order $p^{2}$, then $H$ is $s$-permutable in $G$.

Let $H$ be a subgroup of $P$ of order $p^{2}$. By the hypothesis, $H$ is weakly $s$ semipermutable in $G$. Then there is a subgroup $T$ of $G$ such that $G=H T$ and $H \cap T$ is $s$-semipermutable in $G$. Hence $P=P \cap G=P \cap H T=H(P \cap T)$. Since $P / \Phi(P)$ is abelian, we have $(P \cap T) \Phi(P) / \Phi(P) \triangleleft G / \Phi(P)$. By step (2) (ii), $P \cap T \leq \Phi(P)$ or $P=(P \cap T) \Phi(P)=P \cap T$. If $P \cap T \leq \Phi(P)$, then $H=P \triangleleft G$. In this case, $H$ is s-permutable in $G$. If $P=P \cap T$, then $T=G$ and so $H$ is $s$-semipermutable in $G$. Since $H \leq P=O_{p}(G), H$ is $s$-permutable in $G$ by Lemma 2.1 .

(4) There exists a subgroup $H$ of $P$ such that $|H|=p^{2}$ which is not contained in $\Phi(P)$.

If $\Phi(P)=1$, then it is clear. Hence we may assume that $\Phi(P) \neq 1$. If $|P|=p^{3}$, then clearly $P$ has a maximal subgroup of order $p^{2}$. Since $P$ is not cyclic by Burnside's Theorem [11, Theorem 4.3, P.252], $P$ has at least two 
different maximal subgroups $P_{1}$ and $P_{2}$. If $P_{1}$ and $P_{2}$ are all contained in $\Phi(P)$, then $P=P_{1} P_{2} \leq \Phi(P)$, a contradiction. Hence, we can assume that $|P|>p^{3}$. Let $x \in P \backslash \Phi(P)$ and $a \in \Phi(P)$ where $|a|=p$. Since $\Phi(P) \leq Z(P)$, $\langle x\rangle\langle a\rangle \leq G$. By Step (2), we see that $|x|=p$ or 4 . If $|x|=4$, we can choose $H=\langle x\rangle$. If $|x|=p$, then $|\langle x\rangle\langle a\rangle| \leqslant p^{2}$. If $|\langle x\rangle\langle a\rangle|=p$, then $\langle x\rangle=\langle a\rangle$, a contradiction. Hence $|\langle x\rangle\langle a\rangle|=p^{2}$. Therefore (4) holds.

(5) Final contradiction.

By Step (2), $G=[P] Q$. By Step (4), there exists a subgroup $H$ of $P$ with order $p^{2}$ such that $H \not \leq \Phi(P)$. Then by (3), $H$ is $s$-permutable in $G$. Hence $H Q=Q H$. Then $H=H(Q \cap P)=H Q \cap P \triangleleft H Q$. It follows that $Q \leq N_{G}(H)$. On the other hand, since $P / \Phi(P)$ is abelian, $H \Phi(P) / \Phi(P) \triangleleft P / \Phi(P)$. This implies that $H \Phi(P) / \Phi(P) \triangleleft G / \Phi(P)$. However, since $P / \Phi(P)$ is chief factor of $G$, we obtain that $H \Phi(P)=P$ and consequently $H=P$, a contradiction.

THEOREM 3.23. Let $\mathcal{F}$ be a saturated formation containing $\mathcal{U}$, the class of all supersolvable groups. Suppose that $G$ is a group with a solvable normal subgroup $H$ such that $G / H \in \mathcal{F}$. If all maximal subgroups of all Sylow subgroups of $F(H)$ are weakly s-semipermutable in $G$, then $G \in \mathcal{F}$.

Proof. Assume that the assertion is false and let $(G, H)$ be a counter example with $|G||H|$ is minimal. Let $P$ be an arbitrary Sylow $p$-subgroup of $F(H)$. Clearly $P \triangleleft G$. We proceed the proof by the following steps.

(1) $P \cap \Phi(G)=1$.

If $P \cap \Phi(G) \neq 1$, then $P \cap \Phi(G)=R \triangleleft G$. Obviously, $(G / R) /(H / R) \cong$ $G / H \in \mathcal{F}$ and $F(H / R)=F(H) / R$. Let $P_{1} / R$ be a maximal subgroup of the Sylow $p$-subgroup $P / R$. Then $P_{1}$ is a maximal subgroup of the Sylow $p$-subgroup $P$. By hypothesis, $P_{1}$ is weakly $s$-semipermutable in $G$. Hence $P_{1} / R$ is weakly $s$-semipermutable in $G / R$ by Lemma 2.2 . Let $M_{1} / R$ be a maximal subgroup of the Sylow $q$-subgroup of $F(H) / R$, where $p \neq q$. It is clear that $M_{1}=Q_{1} R$, where $Q_{1}$ is a maximal subgroup of the Sylow $q$ subgroup of $F(H)$. Then $Q_{1}$ is weakly $s$-semipermutable in $G$ by hypothesis. Hence $M_{1} / R$ is weakly $s$-semipermutable in $G / R$ by Lemma 2.2. Now we have proved that $(G / R, H / R)$ satisfies the hypotheses of the theorem. Therefore $G / R \in \mathcal{F}$ by minimal choice of $(G, H)$. Since $R \leq \Phi(G)$ and $\mathcal{F}$ is a saturated formation, we have that $G \in \mathcal{F}$, a contradiction. Thus (1) holds.

(2) $P=R_{1} \times R_{2} \times \cdots \times R_{m}$, where $R_{i}(i=1,2, \cdots, m)$ is some normal subgroup of $G$ of order $p$. 
Since $P \triangleleft G$ and $P \cap \Phi(G)=1, \quad P=R_{1} \times R_{2} \times \cdots \times R_{m}$, where $R_{i}(i=1,2, \cdots, m$ is an abelian minimal normal subgroup of $G$ by Lemma 2.3. We now prove that $\left|R_{i}\right|=p$. Since $R_{i} \nsubseteq \Phi \Phi(G)$, there exists a maximal subgroup $M$ of $G$ such that $G=R_{i} M$ and $R_{i} \cap M=1$. Let $M_{p}$ be a Sylow $p$ subgroup of $M$ and $G_{p}=M_{p} R_{i}$. Then $G_{p}$ is a Sylow $p$-subgroup of $G$. Let $G_{1}$ be a maximal subgroup of $G_{p}$ containing $M_{p}$ and $P_{1}=G_{1} \cap P$. Then $\left|P: P_{1}\right|=\left|P: G_{1} \cap P\right|=\left|P G_{1}: G_{1}\right|=\left|G_{p}: G_{1}\right|=p$ and so $P_{1}$ is a maximal subgroup of $P$. We also have that $P_{1} M_{p}=\left(G_{1} \cap P\right) M_{p}=G_{1} \cap P M_{p}=$ $G_{1} \cap G_{p}=G_{1}$ and $P_{1} \cap M_{p}=P \cap G_{1} \cap M=P \cap M_{p}$. By hypothesis, $P_{1}$ is weakly $s$-semipermutable in $G$. Hence there exists a subgroup $T$ of $G$ such that $G=P_{1} T$ and $P_{1} \cap T$ is $s$-semipermutable in $G$. By Lemma 2.1(c), $P_{1} \cap T$ is $s$-permutable in $G$. Then, for an arbitrary Sylow $q$-subgroup $G_{q}$ of $G$ with $q \neq p,\left(P_{1} \cap T\right) G_{q}=G_{q}\left(P_{1} \cap T\right)$. Hence $P_{1} \cap T=\left(P_{1} \cap T\right)\left(P \cap G_{q}\right)=$ $P \cap\left(P_{1} \cap T\right) G_{q} \triangleleft\left(P_{1} \cap T\right) G_{q}$. It follows that $G_{q} \leq N_{G}\left(P_{1} \cap T\right)$. On the other hand, $P \cap T \triangleleft T$ and $P \cap T \triangleleft P$ since $P$ is abelian. Hence $P \cap T \triangleleft P T=G$ and consequently $P_{1} \cap T=G_{1} \cap P \cap T \triangleleft G_{1}$. It follows that $P_{1} \cap T \triangleleft G_{1} P=G_{p}$. This shows that both $G_{p}$ and $G_{q}$ are contained in $N_{G}\left(P_{1} \cap T\right)$. The arbitrary choice of $q$ implies that $P_{1} \cap T \triangleleft G$ and so $P_{1} \cap T \leq\left(P_{1}\right)_{G}$. Assume that $P_{1} \cap T<\left(P_{1}\right)_{G}$ and let $N=\left(P_{1}\right)_{G} T$. Then $G=P_{1} T=P_{1}\left(P_{1}\right)_{G} T=P_{1} N$ and $P_{1} \cap N=P_{1} \cap\left(P_{1}\right)_{G} T=\left(P_{1}\right)_{G}\left(P_{1} \cap T\right)=\left(P_{1}\right)_{G}$. This shows that there always exists a subgroup $K$ of $G$ such that $G=P_{1} K$ and $P_{1} \cap K=\left(P_{1}\right)_{G}$.

Since $P$ is abelian, $P_{1}(P \cap M) \triangleleft P$. Thus $P_{1}(P \cap M)=P$ or $P_{1}(P \cap M)=$ $P_{1}$. If $P_{1}(P \cap M)=P$, then $G=P M=P_{1}(P \cap M) M=P_{1} M$ and so $P=P \cap P_{1} M=P_{1}(P \cap M)=P_{1}\left(P \cap G_{1} \cap M\right)=P_{1}\left(P_{1} \cap M\right)=P_{1}$, a contradiction. Hence $P_{1}(P \cap M)=P_{1}$ and so $P \cap M \leq P_{1}$. Since $P \cap M \triangleleft G$ by Lemma $2.4, P \cap M \leq\left(P_{1}\right)_{G}=P_{1} \cap K$.

Assume that $K<G$. Let $K_{1}$ be a maximal subgroup of $G$ containing $K$. Then $P \cap K_{1} \triangleleft G$ by Lemma 2.4. Hence $\left(P \cap K_{1}\right) M$ is a subgroup of $G$. Since $M<G,\left(P \cap K_{1}\right) M=G$ or $\left(P \cap K_{1}\right) M=M$. If $\left(P \cap K_{1}\right) M=G=P M$, then $P=P \cap\left(P \cap K_{1}\right) M=\left(P \cap K_{1}\right)(P \cap M)=P \cap K_{1}$ since $P \cap M \leq\left(P_{1}\right)_{G}=$ $P_{1} \cap K \leq P \cap K_{1}$. It follows that $P \leq K_{1}$ and hence $G=P K \leq P K_{1}=K_{1}$, a contradiction. If $\left(P \cap K_{1}\right) M=M$, then $P \cap K_{1} \leq M$ and so $P_{1} \cap K \leq$ $P \cap K \leq P \cap K_{1}=P \cap K_{1} \cap M \leq P \cap M \leq P_{1} \cap K$. Hence $P_{1} \cap K=P \cap K$. Since $G=P K=P_{1} K,|G: P|=|P K: P|=|K:(P \cap K)|=\left|K:\left(P_{1} \cap K\right)\right|=$ $\left|P_{1} K: P_{1}\right|=\left|G: P_{1}\right|$, which is impossible. Thus $K=G$. It follows that $P_{1} \cap K=P_{1}=\left(P_{1}\right)_{G} \triangleleft G$. Consequently, $P_{1} \cap R_{i} \triangleleft G$. But since $G_{p}=R_{i} M_{p}=$ $R_{i} G_{1}$ and $G_{1}$ is a maximal subgroup of $G_{p}$ containing $M_{p}$, we have $R_{i} \not \leq P_{1}=$ $G_{1} \cap P$. The minimal normality of $R_{i}$ implies that $P_{1} \cap R_{i}=1$. Hence $\left|R_{i}\right|=\left|R_{i}:\left(P_{1} \cap R_{i}\right)\right|=\left|R_{i} P_{1}: P_{1}\right|=\left|R_{i}\left(P \cap G_{1}\right): P_{1}\right|=\left|\left(P \cap R_{i} G_{1}\right): P_{1}\right|=$ $\left|P \cap G_{p}: P_{1}\right|=\left|P: P_{1}\right|=p$. Therefore $R_{i}$ is a cyclic group of order $p$. 
(3) Final contradiction.

Let $R_{i} \subseteq H$ and $C_{0}=C_{H}\left(R_{i}\right)$. We claim that the hypothesis holds for $\left(G / R_{i}, C_{0} / R_{i}\right)$. Indeed, since $G / C_{G}\left(R_{i}\right) \leq \operatorname{Aut}\left(R_{i}\right)$ is abelian, $G / C_{G}\left(R_{i}\right) \in \mathcal{F}$. Consequently, $G / C_{0}=G /\left(H \cap C_{G}\left(R_{i}\right)\right) \in \mathcal{F}$. Besides, since $R_{i} \leq Z\left(C_{0}\right)$ and $F(H) \leq C_{0}$, we have $F(H)=F\left(C_{0}\right)$. Thus $F\left(C_{0} / R_{i}\right)=F(H) / R_{i}$. Let $P / R_{i}$ be a Sylow $p$-subgroup of $F(H) / R_{i}$, where $P$ is a Sylow $p$-subgroup of $F(H)$ and $G_{1} / R_{i}$ is a maximal subgroup of $P / R_{i}$. Then $P_{1}$ is a maximal subgroup of $P$. By hypothesis, $P_{1}$ is weakly $s$-semipermutable in $G$. Hence $P_{1} / R_{i}$ is weakly $s$ semipermutable in $G / R_{i}$ by Lemma 2.2. Now assume that $Q R_{i} / R_{i}$ is the Sylow $q$-subgroup of $F(H) / R_{i}$, where $q \neq p$ and $Q$ is the Sylow $q$ subgroup of $F(H)$. Then every maximal subgroup of $Q R_{i} / R_{i}$ is of the form of $Q_{1} R_{i} / R_{i}$, where $Q_{1}$ is a maximal subgroup of $Q$. By hypothesis and Lemma 2.2, we see that $Q_{1} R_{i} / R_{i}$ is weakly $s$-semipermutable in $G / R_{i}$. This shows that $\left(G / R_{i}, C_{0} / R_{i}\right)$ satisfies the condition of the theorem. The minimal choice of $(G, H)$ implies that $G \in \mathcal{F}$ by Lemma 2.5. The final contradiction completes the proof.

CoRollary 3.24 ([5], Theorem 2). Let $\mathcal{F}$ be a saturated formation containing $\mathcal{U}$, the class of all supersolvable groups. Suppose that $G$ is a group with a solvable normal subgroup $H$ such that $G / H \in \mathcal{F}$. If all maximal subgroups of all Sylow subgroups of $F(H)$ are s-semipermutable in $G$, then $G \in \mathcal{F}$.

COROLlary 3.25 ([4], Theorem 1.4). Let $\mathcal{F}$ be a saturated formation containing $\mathcal{U}$. Suppose that $G$ is a solvable group with a normal subgroup $H$ such that $G / H \in \mathcal{F}$. If all maximal subgroups of all Sylow subgroups of $F(H)$ are s-permutable in $G$, then $G \in \mathcal{F}$.

CoRollary 3.26 ([23], Theorem 1). Let $\mathcal{F}$ be a saturated formation containing $\mathcal{U}$, the class of all supersolvable groups. Suppose that $G$ is a group with a solvable normal subgroup $H$ such that $G / H \in \mathcal{F}$. If all maximal subgroups of all Sylow subgroups of $F(H)$ are c-normal in $G$, then $G \in \mathcal{F}$.

CoRollary 3.27 ([7], Theorem 4.5). Let $\mathcal{F}$ be a saturated formation containing $\mathcal{U}$, the class of all supersolvable groups. Suppose that $G$ is a group with a solvable normal subgroup $H$ such that $G / H \in \mathcal{F}$. If all maximal subgroups of all Sylow subgroups of $F(H)$ are c-supplemented in $G$, then $G \in \mathcal{F}$. 
CoROllary 3.28 ([25], Theorem 1.6). Let $\mathcal{F}$ be a saturated formation containing $\mathcal{U}$, the class of all supersolvable groups. Suppose that $G$ is a group with a solvable normal subgroup $H$ such that $G / H \in \mathcal{F}$. If all maximal subgroups of all Sylow subgroups of $F(H)$ are complemented in $G$, then $G \in \mathcal{F}$.

Corollary 3.29 ([22], Theorem 2). Let $G$ be a group and E a solvable normal subgroup of $G$ such that $G / E$ is supersolvable. If all maximal subgroups of the Sylow subgroups of $F(E)$ are c-normal in $G$, then $G$ is supersolvable.

Corollary 3.30 ([28], Theorem 1.2). Suppose that $G$ is a solvable group with a normal subgroup $H$ such that $G / H$ is supersolvable. If all maximal subgroups of every Sylow subgroup of $F(H)$ are complement in $G$, then $G$ is supersolvable.

Acknowledgement. The author would like to thank the referee for his or her helpful comments and suggestions which have improved the original manuscript to its present form.

\section{REFERENCES}

[1] B. Huppert, Endliche Gruppen I, Springer-Verlag, Berlin-New York, 1967.

[2] O. H. KEGEL, Sylow Gruppen and subnormalteiler endlicher Gruppen, Math. Z., 78 (1962), pp. 205-221.

[3] M. AsAaD - M. RAMADAN - A. SHAALAN, The influence of $\pi$-quasinormality of maximal subgroups of Sylow subgroups of Fitting subgroups of a finite group, Arch. Math., 56 (1991), pp. 521-527.

[4] M. ASAAD, On maximal subgroups of finite group, Comm. Algebre, 26 (1998), pp. 3647-3652.

[5] Q. ZHANG - L. WANG, The infuence of s-semipermutable subgroups on the structure of a finite group, Acta Math. Sin., 48 (2005), pp. 81-88.

[6] Y. WANG, Finite groups with some subgroups of Sylow subgroups c-supplemented, J. Algebra, 224 (2000), pp. 467-478.

[7] Y. WANG - H. WEI - Y. LI, A generalization of Kramer's theorem and its application, Bull. Austral. Math. Soc., 65 (2002), pp. 467-475.

[8] X. Guo - K. P. Shum, Cover-avoidance properties and structure of finite groups, J. Pure Appl. Algebra, 181 (2003), pp. 297-308.

[9] A. N. SkIBA, On weakly s-permutable subgroups of finite groups, J. Algebra, 315 (2007), pp. 192-209.

[10] W. Guo, The theory of classes of groups, Science Press-Kluwer Academic Publishers, Beijing-Boston, 2000. 
[11] D. Gorenstein, Finite Groups, Harper and Row Publishers, New York, 1968.

[12] K. Doenk - T. Hawkes, Finite solvable Groups, de Gruyter, Berlin-New York, 1992.

[13] A. Ballester-Bolinches, $\mathcal{H}$-normalizers and local definitions of saturated formations of finite groups, Israel J. Math., 67 (1989), 312-326.

[14] A. Ballester-Bolinches - Y. WANG, Finite groups with some c-normal minimal subgroups, J. Pure Appl. Algebra, 153 (2000), pp. 121-127.

[15] Y. WANG, c-normality of groups and its properties, J. Algebra, 180 (1996), pp. 954-965.

[16] A. Ballester-Bolinches - Y. WANG - X. Guo, c-supplemented subgroups of finite groups, Glasgow Math. J., 42 (2000), pp. 383-389.

[17] M. Asaad - A. Ballester-Bolinches - M. C. Pedraza-Aguilera, A note on minimal subgroups of finite groups, Comm. Algebra, 24 (1996), pp. 27712776.

[18] Y. WANG - Y. LI - J. WANG, Finite groups with c-supplemented minimal subgroups, Algebra Colloq., 10 (2003), pp. 413-425.

[19] X. ZHong - S. LI, On c-supplemented minimal subgroups of finite groups, Southeast Asian Bull. Math., 28 (2004), pp. 1141-1148.

[20] Y. WANG, The influence of minimal subgroups on the structure of finite groups, Acta Math. Sin., 16 (2000), pp. 63-70.

[21] M. Ramadan - M. Ezzat-Mohamed - A. A. Heliel, On c-normality of certain subgroups of prime power order of finite groups, Arch. Math., 85 (2005), pp. 203-210.

[22] D. LI - X. Guo, The influence of c-normality of subgroups on the structure of finite groups II, Comm. Algebra, 26 (1998), pp. 1913-1922.

[23] H. WEI, On c-normal maximal and minimal subgroups of Sylow subgroups of finite groups, Comm. Algebra, 29 (2001), pp. 2193-2200.

[24] Y. LI, Some notes on the minimal subgroups of Fitting subgroups of finite groups, J. Pure Appl. Algebra, 171 (2002), pp. 289-294.

[25] X. Guo - K. P. SHum, Complementarity of subgroups and the structure of finite groups, Algebra Colloq., 13 (2006), pp. 9-16.

[26] M. RAMADAN, Infuence of normality on maximal subgroups of Sylow subgroups of finite groups, Acta Math. Hungar, 73 (1996), pp. 335-342.

[27] M. AsAAD - P. Csorgo, The infiuence of minimal subgroups on the structure of finite groups, Arch. Math., 72 (1999), pp. 401-404.

[28] D. Li - X. Guo, On complemented subgroups of finite groups, Chinese Ann. Math. Ser. B, 22 (2001), pp. 249-254.

Manoscritto pervenuto in redazione il 9 novembre 2010. 